\title{
Anisotropy of the acoustic emission signal during scratch testing of a single crystal of aluminum
}

\author{
A. V. Danyuk ${ }^{\dagger, 1}$, M. A. Afanasiev ${ }^{1}$, D. L. Merson ${ }^{1}$, A. Y. Vinogradov ${ }^{1,2}$ \\ †alexey.danyuk@gmail.com \\ ${ }^{1}$ Togliatti State University, 14 Belorusskaya St., Togliatti, 445020, Russia \\ ${ }^{2}$ Department of Engineering Design and Materials, Norwegian University of Science and Technology (NTNU), NO-7491 \\ Trondheim, Norway
}

In modern materials science, one of the main directions of solving the problem of improving the performance of parts and mechanisms is a radical improvement in the properties of material surface through its modification or application of coatings. To control the mechanical properties of structurally modified layers and coatings, local test methods are becoming increasingly common. Indentation and scratch testing are the most popular among such local techniques. To increase the significance of these tests, it is plausible to combine them with the registration of the acoustic emission (AE) signal. The purpose of this work is to assess the sensitivity of the AE parameters to the change in the dislocation slip systems using an aluminum single crystal as a test piece. The sample surface has been polished and analyzed by scanning electron microscopy and EBSD. The localized deformation test has been carried out on an instrumented tribometer by moving a conical indenter around the circle with a radius of $100 \mu \mathrm{m}$ under a constant load of $2 \mathrm{~N}$. The advantage of such a test is its ability to plastically deform the surface continuously in all crystallographic directions, preserve the stationary conditions for external factors and ensure the optimal signal-to-noise ratio for recording the AE signal. It is established that, depending on the direction of scratching, the hardness and the AE power exhibit opposite trends, both following the anisotropic properties of a cubic crystal lattice, while changing the configuration of active slip systems can be monitored using an interferometer according to characteristic traces of the slip lines on the polished surface of the sample near epy trace indenter. It is shown that the direction of scratching affects not only the energy of the AE signal, but also its spectral characteristics, in particular, the median frequency, and the extremes in the AE power diagram do not coincide with the extremes in the median frequency diagram.

Keywords: acoustic emission, scratch test, dislocation slip, anisotropy, single crystals.

УДК: $620.179,620.111 .3$

\section{Анизотропия сигнала акустической эмиссии при царапании монокристалла алюминия}

\author{
Данюк А. В. ${ }^{\dagger,}$, Афанасьев М. А. ${ }^{1}$, Мерсон Д. Л. ${ }^{1}$, Виноградов А. Ю. ${ }^{1,2}$ \\ †alexey.danyuk@gmail.com
}

\footnotetext{
${ }^{1}$ Тольяттинский государственный университет, НИИПТ, ул. Белорусская, 14, Тольятти, 445020, Россия

${ }^{2}$ Department of Engineering Design and Materials, Norwegian University of Science and Technology (NTNU), NO-7491

Trondheim, Norway
}

В современном материаловедении одним из основных направлений решения задачи по повышению работоспособности деталей и механизмов является кардинальное улучшение свойств поверхности материалов путем ее модификации или нанесения покрытий. Для контроля механических свойств таких модифицированных слоев и покрытий все большее применение находят методы локальных испытаний: индентирование и склерометрия. Для повышения информативности этих испытаний целесообразно их совмещать с регистрацией акустической эмиссии (АЭ). Целью настоящей работы является проверка чувствительности метода АЭ к изменению направления действующих 
систем скольжения на примере монокристалла алюминия. Поверхность образца предварительно была отполирована механическим способом и проанализирована методом сканирующей электронной микроскопии и EBSD. Локализованная деформация осуществлялась на трибометре путем движения по окружности конического индентора с радиусом при вершине 100 мкм под постоянной нагрузкой 2 Н. Преимуществом такого испытания является возможность за один тест осуществить царапание поверхности в различных кристаллографических направлениях, соблюсти условия стационарности внешних факторов и обеспечить наиболее оптимальное соотношение сигнал/шум для регистрации непрерывного сигнала АЭ. Установлено, что твердость материала при царапании и мощность сигнала АЭ в зависимости от направления царапания ведут себя антибатно и проявляют анизотропию, характерную для кубической кристаллической решетки, при этом изменение конфигурации активных систем скольжения контролировалось оптическим интерферометром белого света по характерным следам выхода линий скольжения на полированной поверхности образца вблизи следа индентора. Показано, что направление царапания влияет не только на мощность сигнала АЭ, но и на его спектральные характеристики, в частности на медианную частоту, причем экстремумы на диаграмме мощности АЭ не совпадают с экстремумами на диаграмме медианной частоты.

Ключевые слова: акустическая эмиссия, царапание, дислокационное скольжение, анизотропия, монокристалл.

В настоящее время во всем мире особое внимание уделяется дизайну поверхностных слоев конструкционных и функциональных материалов, т. к. именно они первыми принимают на себя удар, связанный с внешними воздействиями: силовым, фрикционным, температурным, от агрессивной среды и т. п. Для снижения их пагубного влияния предпринимаются всевозможные способы модификации поверхности как с помощью легирования, так и путем специального структурирования, в том числе за счет перевода материала в ультрадисперсное или нанокристаллическое состояние $[1,2]$. Кроме того, общемировые тенденции к миниатюризации и рациональному расходованию ресурсов стимулируют создание все новых и новых материалов в виде пленок и покрытий $[3,4]$. В связи с этим, возникает необходимость объективной оценки физико-механических свойств поверхностных слоев, покрытий и пленок как на стадии разработки технологий их получения, так и для проверки их изменения в процессе эксплуатации изделий.

Как следствие, за последние десятилетия стали бурно развиваться особые методы микромеханических испытаний: индентирование (вдавливание) и склерометрия (царапание или скретч тесты), которые обладают высокой точностью и воспроизводимостью определения механических параметров, позволяют детально изучать локальные свойства и исследовать локализованную деформацию и механизмы разрушения широкого спектра материалов в микро- и нано- масштабах $[4,5]$.

В указанных видах испытаний рабочими инструментами являются инденторы стандартных форм: пирамидальной, сферической или конической. Современная аппаратура позволяет устанавливать в процессе испытания параметры сопротивления материала вынужденной деформации под индентором за счет регистрации приложенных к инструменту сил (вертикальной и горизонтальной) и перемещения. Анализ поведения нагрузок в процессе внедрения или перемещения индентора (так называемая диаграмма вдавливания или скретч тест) позволяет оценивать особенности деформирования исследуемого материала в нано- и микро- объемах $[6,7,8]$. Для более детального анализа локализованной деформации, созданной индентором, чаще всего проводят микроскопические исследования следа (отпечатка или царапины) при помощи различной техники: оптической, электронной или атомносиловой. Однако, несмотря на высокое пространственное разрешение рельефа, эти методы имеют существенный недостаток: в большинстве случаев они отображают состояние материала на момент окончания испытания, т. е. не позволяют фиксировать деформационный процесс непосредственно в ходе нагружения.

Локализованная деформация под индентором формируется градиентным полем механического напряжения, под действием которого в металлических материалах происходит быстрое скольжение дислокаций - основного элементарного деформационного механизма кристаллических материалов. Идентификация единичных событий дислокационного скольжения является трудной задачей для современной техники, т.к. требует высокого разрешения как в пространстве, так и во времени. Тем не менее, успешные примеры решения этой задачи уже существуют: с помощью применения электронной микроскопии высокого разрешения и метода акустической эмиссии (АЭ) $[9,10]$. Электронная микроскопия с применением механических манипуляторов позволяет регистрировать картину в динамике, но при этом временное разрешение все еще не достаточно для детального изучения деформации при индентировании, а необходимость расширения зоны сканирования, вызванная постоянным движением области деформации, приводит к ухудшению пространственной детализации. Альтернативный метод «наблюдения» за деформацией, метод АЭ, постепенно становится доминирующим при микромеханических испытаниях, например, при индентировании [11] или царапании пластичных и хрупких материалов [12], или материалов с покрытием $[13,14]$. Однако и у метода АЭ есть свои проблемы, основной из которых является необходимость регистрация сигналов очень низкой амплитуды, близкой к уровню окружающих шумов, поскольку при локальных схемах нагружения АЭ генерируется исключительно малым объемом деформируемого материала. Вместе с тем, современные программно-аппаратные комплексы позволяют существенно улучшить чувствительность метода за счет использования алгоритмов обработки непрерывного сигнала АЭ [15], благодаря чему был получен ряд новых, чрезвычайно важных результатов. Так, в работе [16] было установлено, 
что при царапании поликристаллического крупнозернистого материала наблюдается локальная нестационарность непрерывного сигнала акустической эмиссии при пересечении индентором границ зерен, при этом спектр плотности мощности сигналов АЭ оказался строго индивидуальным для каждой ориентации зерна. Указанные оптимистичные результаты стимулировали авторов настоящей работы на более детальное исследование зависимости сигналов АЭ от направления царапания по отношению к ориентации кристаллической решетки.

С этой целью для исследования был выбран объемный монокристалл алюминия с площадью поверхности

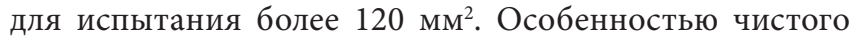
алюминия - классического представителя кристаллов с ГЦК решеткой - является высокая величина энергии дефекта упаковки, благодаря чему в нем при комнатной температуре реализуется исключительно дислокационный механизм деформации (работа систем двойникования исключена). Поверхность для испытания была ориентирована близко к кристаллографической плоскости (001). Подготовка поверхности для испытания выполнена по процедуре подготовки для EBSD (electron back scattered diffraction) анализа микроструктуры: после шлифования на бумаге \#2000 проведена полировка поверхности с применением алмазных суспензий 3 мкм и 1 мкм и финишная полировка с суспензией на основе коллоидного кремния 0.04 мкм длительностью до трех часов. Схематичное изображение кубической ячейки показано в центре Рис. 1. Растровая электронная микроскопия выполнена на микроскопе Zeiss Sigma с детекторами EBSD и EDS EDAX/TSL.

Выбор индентора и траектории его движения ключевые задачи данного испытания. Для регистрации непрерывного сигнала АЭ особенно важно соблюдение условия стационарности, т.е. неизменности внешних параметров эксперимента: шум работы исполнительных механизмов, тепловой и электрический шум аппаратуры, расстояние и среда прохождения АЭ. Наиболее подходящей схемой испытания, удовлетворяющей обозначенным требованиям, является движение индентора по окружности, которое позволяет за одно испытание в компактной области плоской поверхности образца пройти все направления от 0 до 360 градусов. Форма индентора должна обеспечивать хорошую устойчивость под действием боковых сил, возникающих из-за проявления анизотропии действующих систем скольжения, т.е. индентор должен быть центрально симметричной формы - коническим или сферическим. От остроты (радиуса при вершине) индентора зависит глубина внедрения, т.е. деформируемый объем, и, как следствие, средняя скорость деформации, которая обратно пропорциональна ширине следа [17]. С другой стороны, под действием острого индентора существует высокая вероятность перехода процесса пластической деформации в режим резания. Исходя из вышеизложенного и с целью обеспечения оптимального соотношения сигнал/шум для регистрации АЭ была выбрана коническая форма индентора со сферической вершиной радиусом 100 мкм.
Для осуществления скретч-теста (царапания) был собран испытательный комплекс, включающий универсальный трибометр фирмы «Nanovea» и аппаратуру регистрации и анализа непрерывного сигнала акустической эмиссии РАС PCI-2. Нагружение индентора выполняли статическим весом (грузом) величиной $2 \mathrm{H}$, вращение столика с закрепленным образцом проводили на два оборота (720 градусов) монотонно с периодом 20 секунд на один оборот. Положение индентора в начальный момент испытания соответствует точке 0 градусов на Рис. 1. Диаметр окружности деформированного материала по средней линии составлял 4.35 мм, что соответствовало линейной скорости перемещения индентора около 41 мм/мин (683 мкм/с). АЭ регистрировали в непрерывном режиме в течении всего испытания с частотой дискретизации 5 МГц в полосе частот $10 \div 1200$ кГц с использованием широкополосного датчика AE-900S WB (частотный диапазон $100 \div 900$ кГц) при общем усилении сигнала 66 дБ.

След индентора в виде окружности после скретч теста, полученный за два оборота, показан на Рис. 1. Внутри этой окружности в полярных координатах приведена диаграмма твердости, которая обладает хорошо заметной 90-градусной анизотропией, связанной с кристаллографической ориентацией монокристалла, приведенной на врезке в центре Рис. 1.

Непрерывный сигнал АЭ, зарегистрированный за все время испытания, показан на Рис. 2. Представлен-

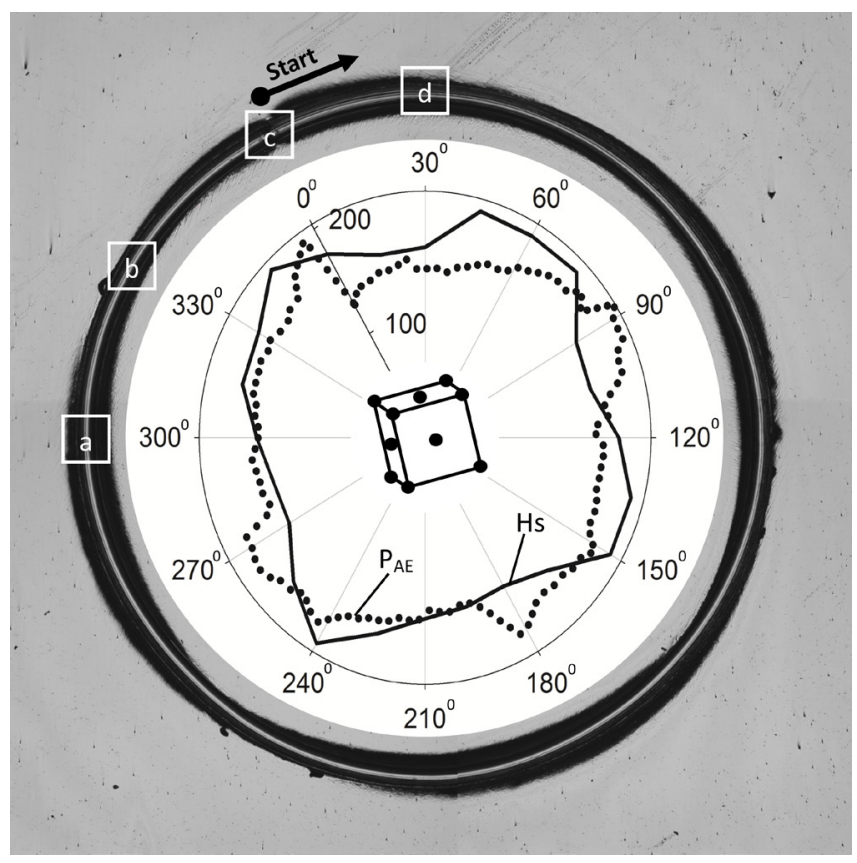

Рис. 1. След от индентора после испытания, ориентация элементарной ячейки монокристалла алюминия (в центре); диаграмма: твердость при царапании HS, MПа (сплошная линия), мощность акустической эмиссии, $10 \times P_{\text {Аэ}}$, мкВ $^{2} / \mathrm{OM}$ (пунктирная линия). a, b, c, d - места расположения кадров, приведенных на Рис. 4.

Fig. 1. Circular scratch after testing (outside), cubic crystal orientation of the aluminum single crystal (center). Polar graph: scratch hardness HS, MPa (solid line); power of acoustic emission, $10 \times P_{\mathrm{AE}}, \mu \mathrm{V}^{2} /$ Ohm (dotted line). a, b, c, d - locations of zoom-in frames for Fig. 4. 
ный фрагмент длительностью около 50 секунд, на котором по уровню регистрируемого сигнала можно хорошо различить все этапы испытания: первый (I) - фоновый шум установки до начала движения, т. е. когда механический привод активен, но находится в режиме тормоза; второй и третий (II и III) - сигнал во время непрерывного монотонного царапания по окружности на 720 градусов с постоянным радиусом (первый и второй оборот соответственно); четвертый (IV) - фоновый шум установки в режиме тормоза после испытания. Для более детального анализа АЭ был выполнен расчет его средней мощности и медианной частоты (частота, делящая площадь под кривой спектра плотности мощности на две равные части) [18] для скользящего интервала длительностью 100 мс, результат которого приведен на диаграмме, синхронизированной с углом поворота индентора во время царапания (Рис. 3). Для удобства анализа, результаты для каждого круга на Рис. 3 приведены друг под другом.

Акустическая эмиссия хорошо демонстрирует ключевые моменты деформации под индентором: на первом обороте деформируется исходная структура монокристалла с активным образованием линий скольжения в неупрочненной области как перед индентором (Рис. 4), так и с боков, поэтому на всем протяжении первого оборота мощность АЭ выше, чем при выполнении второго оборота, когда индентор движется по уже предварительно упрочненному материалу и скорость деформационного упрочнения снижается. Однако непосредственно в момент замыкания траектории первого оборота происходит резкое увеличение мощности АЭ, которое связано с преодолением движущегося впереди индентора фронта локализованной деформации неоднородности поверхности в виде упрочненного фрагмента материала (буртика), сформировавшегося вокруг индентора в стартовой точке.

На Рис. 3 хорошо видно, что изменение каждого из параметров АЭ (мощности и медианной частоты) в зависимости от угла поворота на первом и втором кругах происходит синхронно и закономерно с периодичностью 90 градусов. При этом не обычно, что экстремумы на диаграммах мощности АЭ и медианной частоты не совпадают. Объяснение причины последнего результата, а также ответ на вопрос почему абсолютные

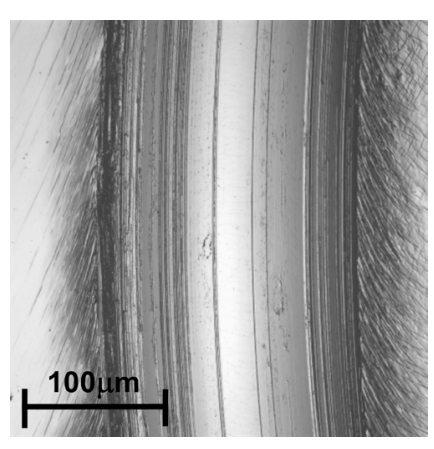

a

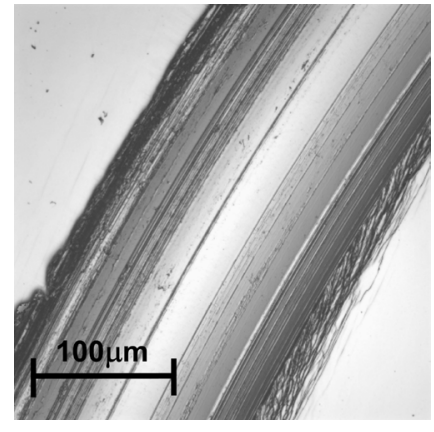

b

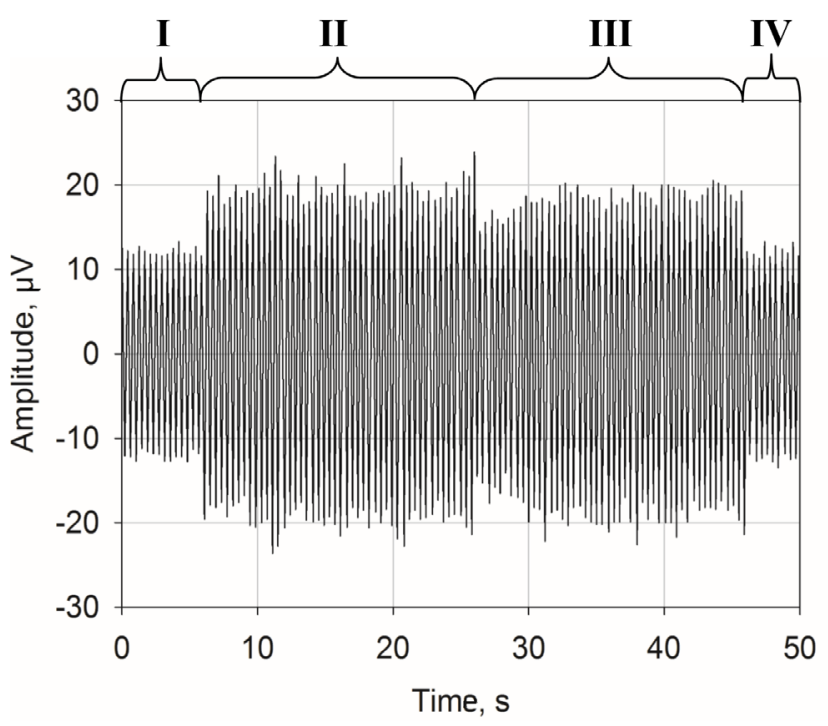

Рис. 2. Акустическая эмиссия при царапании монокристалла алюминия.

Fig. 2. Acoustic emission signal at scratch test of the aluminum single crystal.

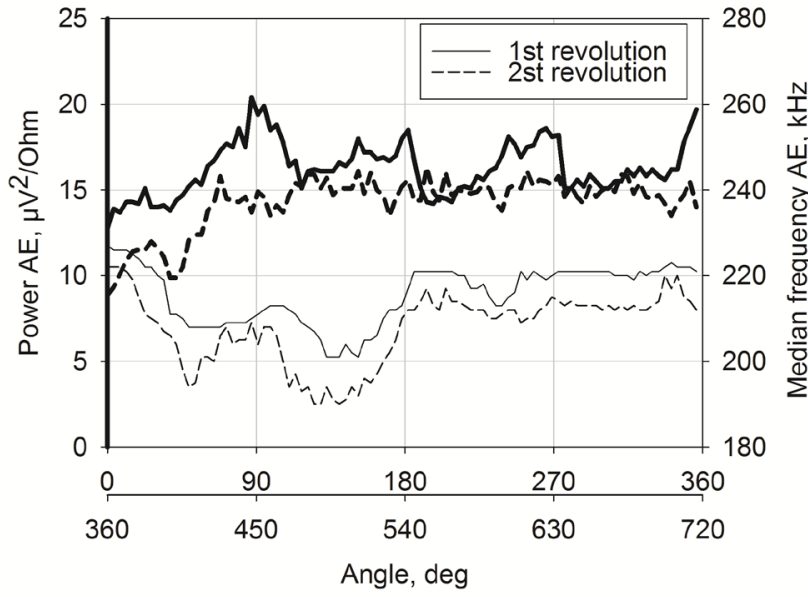

Рис. 3. Мощность акустической эмиссии (жирная линия) и медианная частота (тонкая линия); первый оборот (сплошная линия), второй оборот (пунктирная линия).

Fig. 3. Power of acoustic emission (bold line), median frequency (thin line); $1^{\text {st }}$ revolution at test (solid line), $2^{\text {nd }}$ revolution at test (dashed line).

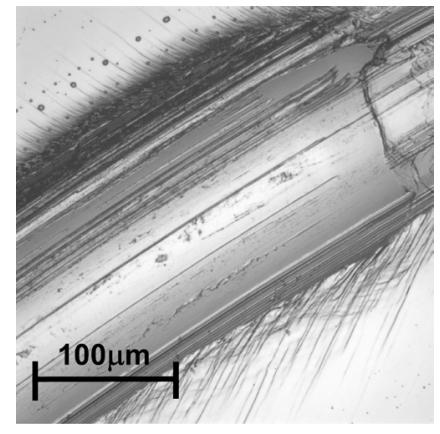

C

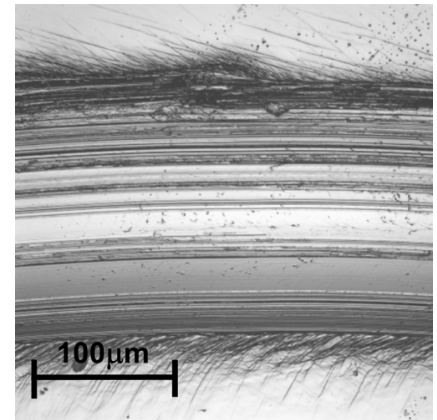

d

Рис. 4. Линии скольжения вблизи следа индентора при различных значениях угла полярной системы координат (a)-(d), отмеченных на Рис. 1.

Fig. 4. Slip lines close to the indenter footprint at different angles (a)-(d) of polar coordinate, see corresponding marks in Fig.1. 
значения медианной частоты на втором круге оказались синхронно ниже по сравнению с первым (Рис. 3), выходят за рамки настоящей статьи и будут обнародованы авторами позднее.

Акустическая эмиссия - это отклик релаксационных процессов перестройки кристаллической решетки, например, вызванных скольжением дислокаций. Активация скольжения дислокаций в кристаллах наступает с достижением локального напряжения, превышающего пороговое значение, которое зависит от взаимной ориентации системы скольжения и направления внешнего напряжения в соответствии с фактором Шмида. Именно поэтому при индентировании и царапании монокристаллов наблюдается анизотропия отпечатка от индентора (форма розетки или ширина царапины) в зависимости от направления движения индентора. Таким образом, от конфигурации схемы нагружения зависят напряжение активации, количество активных систем скольжения [19] и, соответственно, отклик системе в виде АЭ. На Рис. 1 приведены совмещенные диаграммы твердости и мощности АЭ, изменение которых со всей очевидностью происходит антибатно в зависимости от угла оборота: меньшей твердости соответствует более мощная АЭ и наоборот, что совершенно закономерно, т.к. чем меньше твердость, тем легче и в большем объеме деформируется материал и, соответственно, наблюдается большее значение мощности непрерывного сигнала АЭ.

Наиболее очевидным источником АЭ является выход дислокаций на свободную поверхность [20,21], которой в нашем случае служит поверхность царапания. Поэтому в данной работе поверхность образца вблизи следа индентора была дополнительно исследована с применением метода оптической интерферометрии, который позволяет получить высокое разрешение по рельефу поверхности и контрастно идентифицировать следы выхода дислокаций в виде линий скольжения. На Рис. 4 представлена полученная с помощью оптического интерферометра белого света Zygo-9100 (объектив $\times 50$, канал интенсивности) последовательность из четырех снимков с шагом тридцать градусов. Расположение этих снимков на окружности царапания указано на Рис. 1. Обнаруженные на снимках длинные линии скольжения в области малой деформации (на некотором удалении от «навала») могут соответствовать исключительно следам четырех кристаллографических плоскостей $\{111\}$, в которых происходит скольжение. Так же по величине и удаленности от следа индентора в поперечном направлении линий скольжения можно судить о легкости скольжения и соответственно о твердости направления царапания. Так, направления $300^{\circ}$, $0^{\circ}, 30^{\circ}$ (Рис. $\left.4 \mathrm{a}, \mathrm{c}, \mathrm{d}\right)$ - являются относительно «мягкими» с широким следом и линиями скольжения, расходящимися на большое расстояние от следа, а направление $330^{\circ}$ (Рис. 4 b), наоборот, самое «твердое», т. к. следы двух плоскостей скольжения практически совпадают с направлением царапания, а две другие - ему перпендикулярны, что существенно ограничивает скольжение и деформацию, поэтому линии скольжения очень редкие и расположены близко к индентору.

\section{Заключение}

В работе показано, что в процессе кругового царапания с непрерывным изменением конфигурации активных систем скольжения $\{111\}<110>$ в ГЦК решетке монокристалла алюминия наблюдаются параметры непрерывного сигнала акустической эмиссии, изменение которых носит детерминированный характер - при схожих условиях активации систем скольжения регистрируется АЭ со схожими параметрами. Связь между параметрами скольжения (выхода дислокаций на свободную поверхность) и АЭ еще предстоит изучить, но уже сейчас можно предположить, что в качестве ключевого параметра в такой модели будет выступать фактор Шмида, величина которого определяет значение критического напряжения страгивания дислокаций.

Метод АЭ применительно к микромеханическим испытаниям демонстрирует высокую чувствительность, причем не только к интегральным свойствам деформационного процесса (скорость и объем деформируемого материала), но и к дискретным элементарным событиям пластической деформации - движению отдельных дислокаций или небольших групп дислокаций, образующих линии скольжения.

Благодарность/Acknowledgements. Настоящая работа выполнена при финансовой поддержке Министерства науки и образования РФ в рамках проекта по Госзаданию N 11.5281.2017/8.9./This work was carried out with the financial support of the Ministry of Science and Education of the Russian Federation in the framework of the project on the the state task N 11.5281.2017/8.9.

\section{Литература/References}

1. Ed. by S. H. Whang. Nanostructured Metals and Alloys. Woodhead Publishing (2011), 840 p.

2. A. Vinogradov. Advanced Engineering Materials. 17 (12), 1720 (2015). DOI: 10.1002/adem.201500177

3. W.D. Munz, D. B. Lewis, P.E. Hovsepian. Surface Engineering. $17 \quad$ (2), $153 \quad$ (2001). DOI: $10.1179 / 026708401101517656$

4. Yu. I. Golovin. Physics of the Solid State. 50 (15), 2205 (2008). (in Russian) [Ю.И. Головин. Физика твердого тела. 50 (12), 2205 (2008).] DOI: $10.1134 /$ S1063783408120019

5. W. C. Oliver, G. M. Pharr. Journal of Material Reserch. 19 (1), 3 (2011). DOI: 10.1557/jmr.2004.19.1.3

6. R. Sánchez-Martín, M. T. Pérez-Prado, J. Segurado, J. M. Molina-Aldareguia. Acta Materialia. 93, 114 (2015), DOI: 10.1016/j.actamat.2015.04.005

7. V. Jardret, H. Zahouani, J. L. Loubet, T. G. Mathia. Wear. 218 (1), 8 (1998). DOI: 10.1016/S0043-1648(98)00200-2

8. J.A. Williams. Tribology International. 29 (8), 675 (1996). DOI:10.1016/0301-679X(96)00014-X

9. A. Vinogradov, A. Danyuk, V.A. Khonik. Journal of Applied Physics. 113 (15), 153503 (2013). DOI: $10.1063 / 1.4801876$ 
10. A. Vinogradov, D. Orlov, A. Danyuk, Y. Estrin. Materials Science and Engineering A. 621, 243 (2015). DOI: $10.1016 /$ j.msea.2014.10.081

11. N.H. Faisal, R. Ahmed, R.L. Reuben. International Materials Reviews. 56, 98 (2011). DOI: $10.1179 / 1743280410$ Y.0000000004

12. V. Perfilyev, I. Lapsker, A. Laikhtman, L. Rapoport. Tribol. Lett. 65, 24 (2017). DOI: $10.1007 /$ s11249-017-0820-1

13. B. Podgornik, O. Wänstrand. Materials Characterization. 55, 173 (2005). DOI: 10.1016/j.matchar.2005.04.011

14. E. Agletdinov, E. Pomponi, D. Merson, A. Vinogradov. Ultrasonics. 72, 89 (2016). DOI: 10.1016/j.ultras.2016.07.014

15. E. Pomponi, A. Vinogradov, A. Danyuk. Signal Processing. 115, 110 (2015). DOI: 10.1016/j.sigpro.2015.03.016

16. A. Vinogradov, A.V. Danyuk, D.L. Merson,
I.S. Yasnikov. Scripta Materialia. 151, 53 (2018). DOI: $10.1016 /$ j.scriptamat.2018.03.036

17. B. J. Briscoe, E. Pelillo, S. K. Sinha. Polymer Engineering and Science. 36, 2996 (1996). DOI: 10.1002/pen.10702

18. A. Vinogradov, M. Nadtochiy, S. Hashimoto, S. Miura. Material Transactions. JIM. 36, 496 (1995). DOI: $10.2320 /$ matertrans1989.36.496

19. C. A. Brookes, P. Green, D. Tabor. Proceedings of The Royal Society A. Mathematical and Physical Sciences. London (1979). DOI: 10.1098/rspa.1979.0115

20. C.B. Scruby, H.N. G. Wadley, J.J. Hill. Journal of Physics D: Applied Physics. 16 (6), 1069 (1983). DOI: 10.1088/0022-3727/16/6/015

21. D. Merson, M. Nadtochiy, V. Patlan, A. Vinogradov, K. Kitagawa. Materials Science and Engineering A. 234-236, 587 (1997). DOI: $10.1016 /$ S0921-5093(97)00248-7 